Non-sterile L-lysine fermentation using engineered phosphite-grown

\title{
Corynebacterium glutamicum
}

Ming Lei ${ }^{1,2}$, Xiwei Peng ${ }^{1,2}$, Wenjun Sun ${ }^{1,2}$, Di Zhang ${ }^{1,2}$, Zhenyu Wang ${ }^{1,2}$, Zhengjiao Yang ${ }^{1,2}$, Chong Zhang ${ }^{1,2}$, Bin $\mathrm{Yu}^{1,2}$, Huanqing $\mathrm{Niu}^{1,2}$, Hanjie Ying ${ }^{1,2,3}$, Pingkai Ouyang $^{1,2}$, Dong Liu ${ }^{1,2,3^{*}}$, Yong Chen ${ }^{1,2^{*}}$

${ }^{1}$ National Engineering Research Center for Biotechnology, College of Biotechnology and Pharmaceutical Engineering, Nanjing Tech University, Nanjing, China

2 State Key Laboratory of Materials-Oriented Chemical Engineering, College of Biotechnology and Pharmaceutical Engineering, Nanjing Tech University, Nanjing,

China

3 School of Chemical Engineering and Energy, Zhengzhou University, Zhengzhou 450000, China 
Table S1. DNA sequences of genes used in this study.

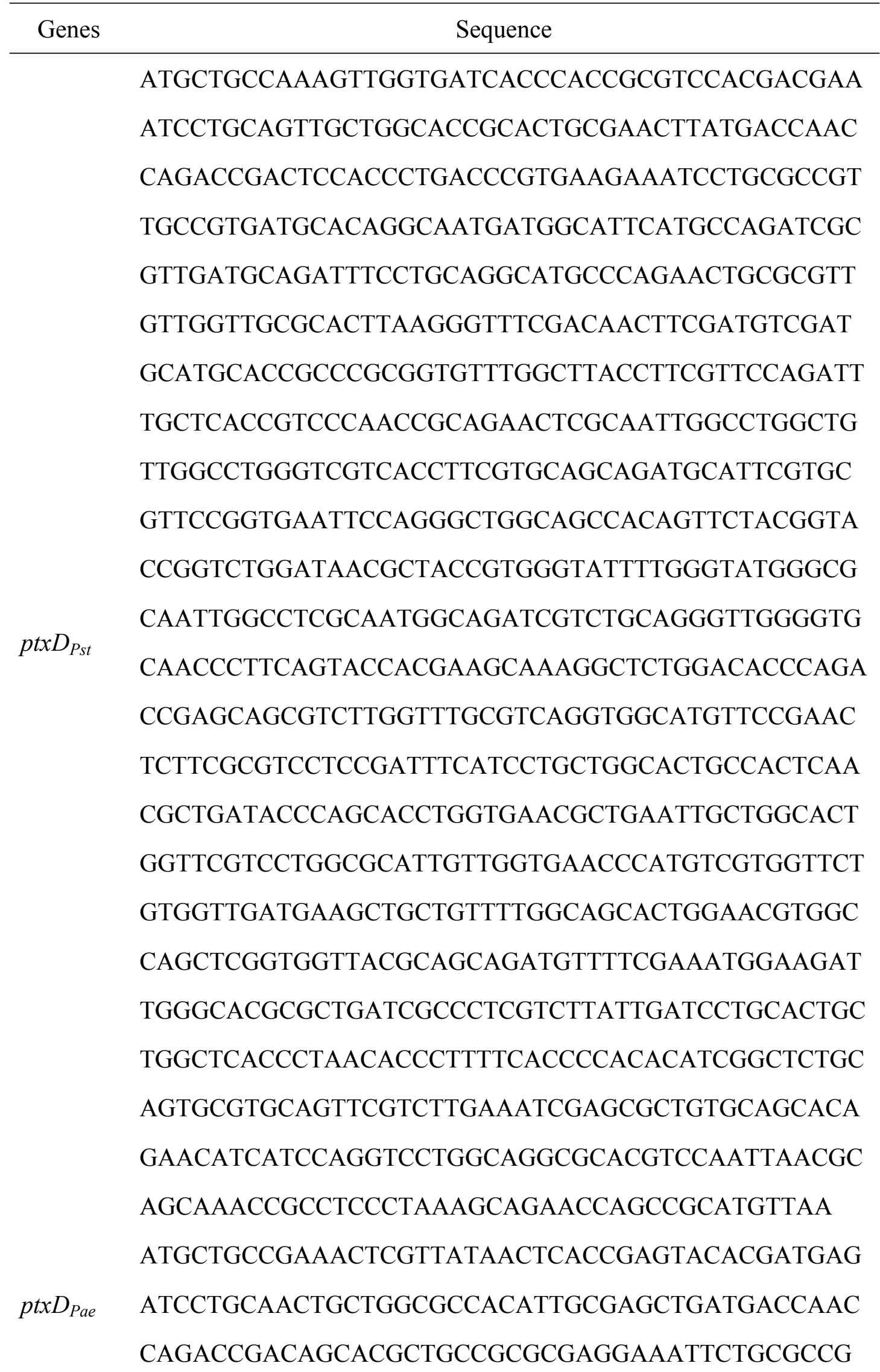


CTGCCGCGATGCTCAGGCGATGATGGCGTTCATGCCCGATCG GGTCGATGCAGACTTTCTTCAAGCCTGCCCTGAGCTGCGTGT AGTCGGCTGCGCGCTCAAGGGCTTCGACAATTTCGATGTGGA CGCCTGTACTGCCCGCGGGGTCTGGCTGACCTTCGTGCCTGA TCTGTTGACGGTCCCGACTGCCGAGCTGGCGATCGGACTGGC GGTGGGGCTGGGGCGGCATCTGCGGGCAGCAGATGCGTTCGT CCGCTCTGGCAAGTTCCAGGGCTGGCAACCACAGTTCTACGG CACGGGGCTGGATAACGCTACGGTCGGCATCCTTGGCATGGG CGCCATCGGACTGGCCATGGCTGATCGCTTGCAGGGATGGGG CGCGACCCTGCAGTACCACGAGGCGAAGGCTCTGGATACAC AAACCGAGCAACGGCTCGGCCTGCGCCGGGTGGCGTGCAGC GAACTCTTCGCCAGCTCGGACTTCATCCTGCTGGCGCTTCCCT TGAATGCCGATACCCAGCATCTGGTCAACGCCGAGCTGCTTG CCCTCGTACGGCCGGGCGCTCTGCTTGTAAACCCCTGTCGTG GTTCGGTAGTGGATGAAGCCGCCGTGCTCGCGGCGCTTGAGC GAGGCCAGCTCGGCGGGTATGCGGCGGATGTATTCGAAATG GAAGACTGGGCTCGCGCGGACCGGCCGCGGCTGATCGATCCT GCGCTGCTCGCGCATCCGAATACGCTGTTCACTCCGCACATA GGGTCGGCAGTGCGCGCGGTGCGCCTGGAGATTGAACGTTGT GCAGCGCAGAACATCATCCAGGCATTGGCAGGTGCGCGCCC AATCAACGCTGCGAACCGTCTGCCCAAGGCCGAGCCTGCCGC ATGTTGA

ATGCTGCCAAAGTTGGTGATCACCCACCGCGTCCACGACGAA ATCCTGCAGTTGCTGGCACCGCACTGCGAACTTGTGACCAAC CAGACCGACTCCACCCTGACCCGTGAAGAAATCCTGCGCCGT $p t x D_{K p n} \quad$ TGCCGTGATGCCCAGGCAATGATGGCATTCATGCCAGATCGC GTGGACGCAGATTTCCTGCAGGCATGTCCAGAACTCCGCGTG GTTGGTTGCGCACTTAAGGGTTTCGATAACTTCGATGTCGAT GCATGCACCGCACGTGGCGTTTGGCTTACCTTCGTGCCAGAT 
TTGCTTACCGTCCCAACCGCAGAACTCGCAATTGGCTTGGCT GTTGGCCTGGGTCGTCACCTTCGTGCAGCAGATGCATTCGTG CGCTCCGGTGAATTCCAGGGTTGGCAGCCACAGTTCTACGGC ACCGGTCTTGATAACGCTACCGTGGGTATTCTGGGCATGGGC GCAATTGGCCTTGCTATGGCAGAACGTCTCCAGGGTTGGGGT GCAACCCTTCAGTACCACGAAGCAAAGGCACTGGACACCCA GACCGAACAGCGTCTTGGTCTCCGTCAGGTGGCATGTTCCGA ACTCTTCGCGTCCTCCGATTTCATCCTGCTGGCGCTTCCACTC AACGCAGATACCCAGCACTTGGTGAACGCAGAACTGCTGGC ACTTGTCCGCCCAGGTGCACTTCTTGTTAACCCATGCCGCGGT TCCGTCGTTGATGAAGCTGCTGTGCTCGCTGCCCTGGAACGT GGTCAGCTTGGTGGTTACGCAGCAGATGTTTTCGAAATGGAA GATTGGGCACGCGCAGATCGCCCACGTCTTATTGATCCAGCA CTGCTGGCACACCCTAACACCTTGTTCACCCCACACATCGGC TCCGCAGTGCGTGCAGTTCGTCTTGAAATTGAGCGCTGTGCA GCACAGAACATCATCCAGGTCCTGGCAGGCGCACGTCCAATT AACGCAGCAAACCGCCTGCCAAAAGCTGAACCAGCAGCATG TTAA

AGATCAGTAGGCGCGTAGGGTAAGTGGGGTAGCGGCTTGTTA GATATCTTGAAATCGGCTTTCAACAGCATTGATTTCGATGTAT TTAGCTGGCCGTTACCCTGCGAATGTCCACAGGGTAGCTGGT

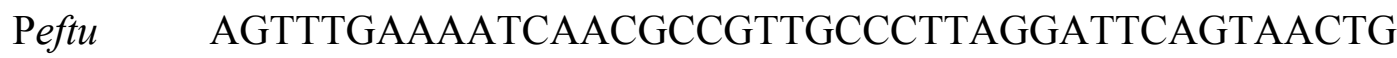
GCACATTTTGTAATGCGCTAGATCTGTGTGCTCAGTCTTCCAG GCTGCTTATCACAGTGAAAGCAAAACCAATTCGTGGCTGCGA AAGTCGTAGCCACCACGAAGTCCAGGAGGACATACA 


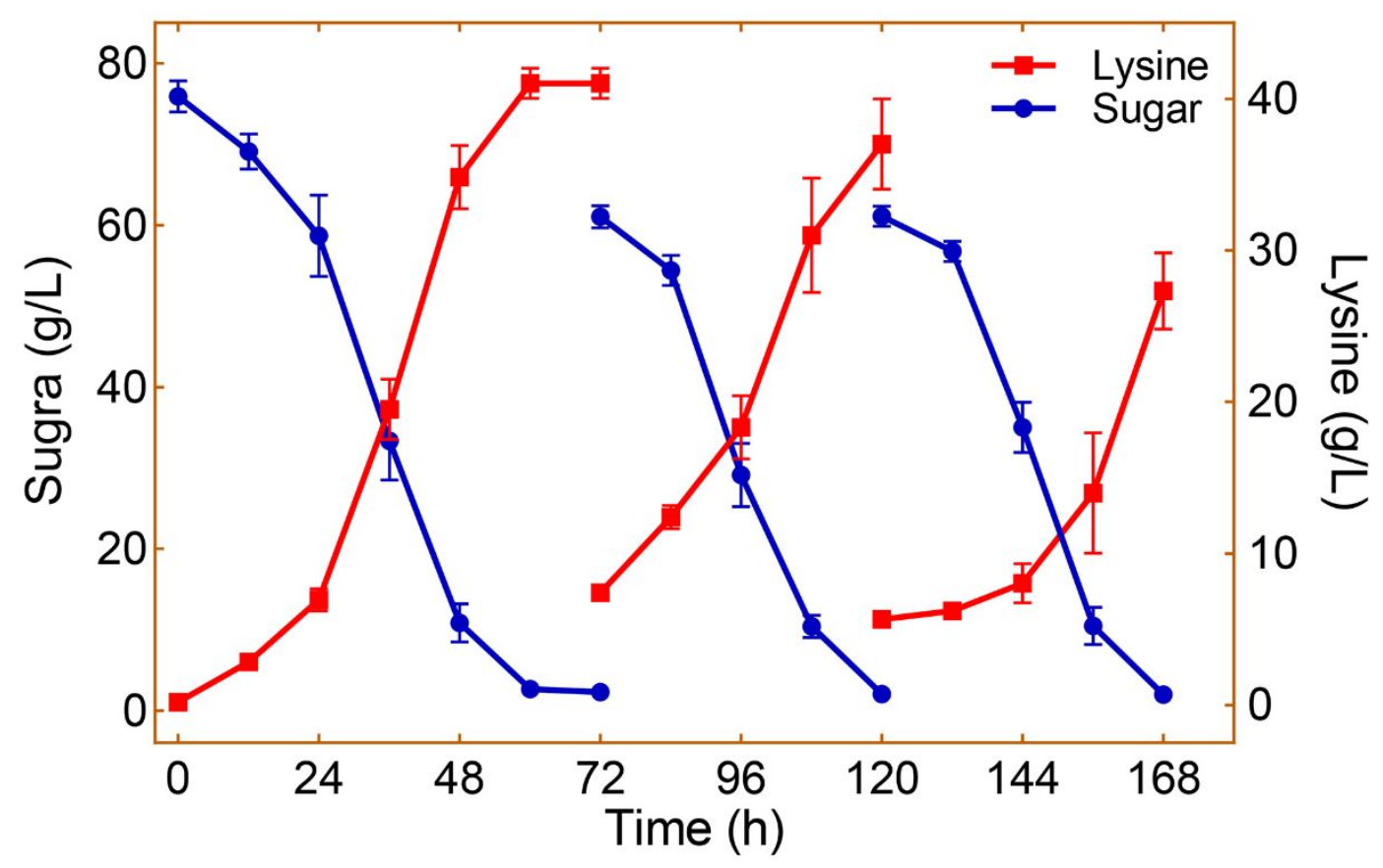

Figure S1. L-lysine production and sugar consumption in repeated batch fermentation by $\mathrm{Cg} \Delta$ exeR*Pst in non-sterile Pt medium. Error bars are given showing standard deviations for $\mathrm{n}=3$.

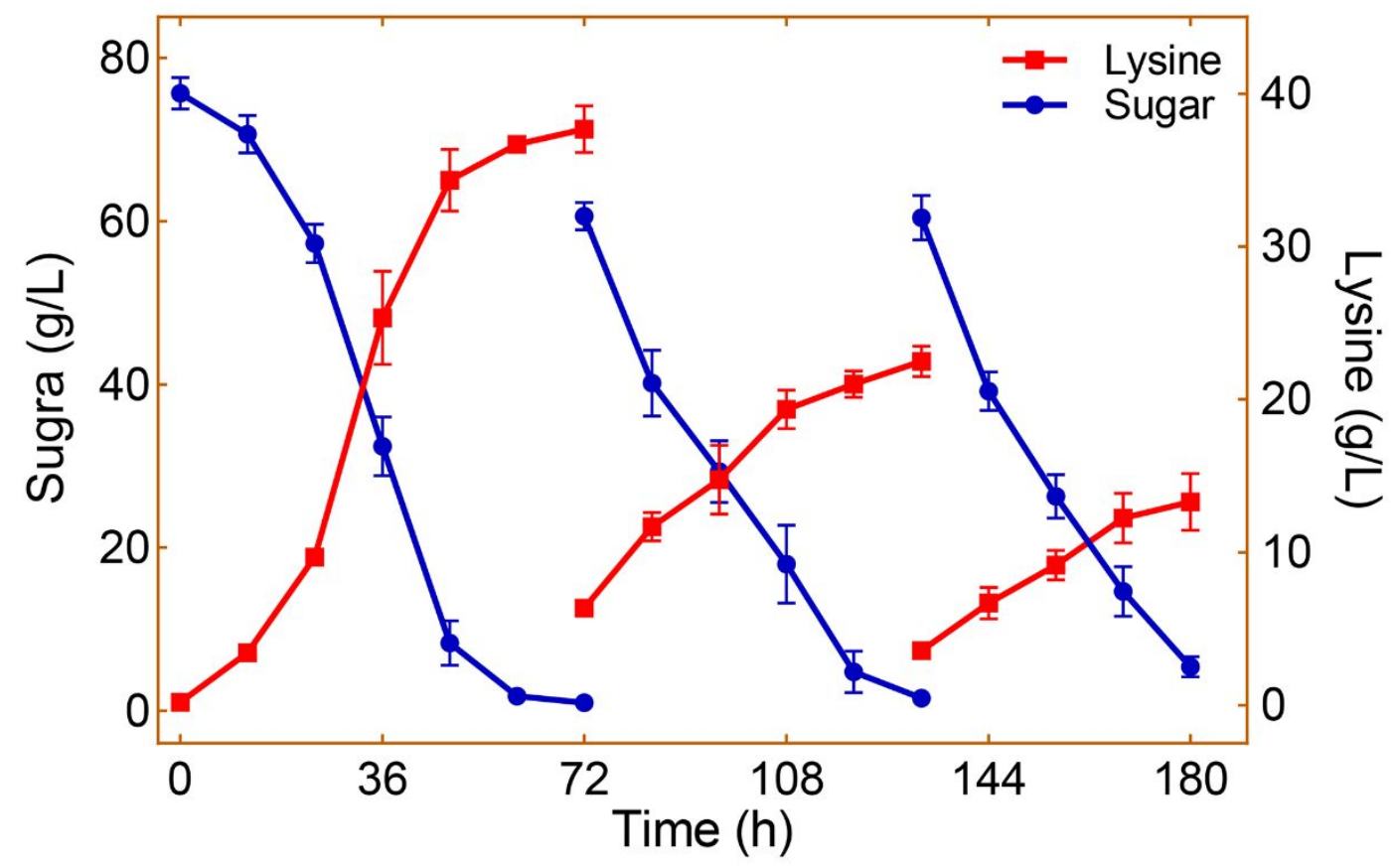


Figure S2. L-lysine production and sugar consumption in immobilized continuous (repetitive batch) fermentation by $\mathrm{Cg} \Delta$ exeR*Pst in non-sterile Pt medium. Error bars are given showing standard deviations for $\mathrm{n}=3$. 